\title{
Fuzzification of Feynman Path Integral and Its Effect on Field Theory and Quantum Gravity-Reformation and Redevelopment of Quantum Theory
}

\author{
Wenbing Qiu \\ Department of Physics, Faculty of Science, Shihezi University, Shihezi, China \\ Email: 2962546794@qq.com
}

How to cite this paper: Qiu, W.B. (2020) Fuzzification of Feynman Path Integral and Its Effect on Field Theory and Quantum Gravity-Reformation and Redevelopment of Quantum Theory. Journal of Modern Physics, 11, 2053-2065.

https://doi.org/10.4236/jmp.2020.1112129

Received: October 9, 2020

Accepted: December 27, 2020

Published: December 30, 2020

Copyright $\odot 2020$ by author(s) and Scientific Research Publishing Inc. This work is licensed under the Creative Commons Attribution International License (CC BY 4.0).

http://creativecommons.org/licenses/by/4.0/

\section{(c) (i) Open Access}

\begin{abstract}
The quantum probability theory of fuzzy event is suggested by using the idea and method of fuzzy mathematics, giving the form of fuzzy event path integral, membership degree amplitude, fuzzy field function, Green function, physical quantity and fuzzy diagram. This theory reforms quantum mechanics, making the later become its special case. This theory breaks unitarity, gauge invariance, probability conservation and information conservation, making these principles become approximate ones under certain conditions. This new theory, which needs no renormalization and can naturally give meaningful results which are in accordance with the experiments, is the proper theory to describe microscopic high-speed phenomenon, whereas quantum mechanics is only a proper theory to describe microscopic low-speed phenomenon. This theory is not divergent under the condition of there being no renormalization and infinitely many offsetting terms, thereby it can become the theoretical framework required for the quantization of gravity.
\end{abstract}

\section{Keywords}

Quantum Probability, Fuzzy Event, Path Integral, Membership Degree, Membership Degree Amplitude, Fuzzy Graph

\section{Introduction}

Since Hawking offered the problem of black hole information loss [1], scholars have tried their best to save the conservation of information [2] [3] [4], because the non conservation of information will destroy the unitarity of quantum theory and bring a major crisis to quantum theory [5]-[12] in which brilliant 
achievements have been made. However, here there exists a problem: quantum theory is a theory to describe reversible process, whereas black hole radiation is irreversible process [13]. How can the former fit describing the latter! The reason why black hole radiation is irreversible is that the heat capacity of black hole is negative and there is no stable heat balance between black hole and the outside world. There must be temperature difference between the black hole and the outside world, and the radiation process must be irreversible. Quantum theory is not suitable for describing irreversible processes, and it is incompatible with the second law of thermodynamics. Thereby, the reformation of quantum theory or a new theory has to be found to depict irreversible black hole radiation. This is the work of this paper.

In my first work [14] on fuzzy quantum probability, it is mentioned that there are two directions for developing quantum probability into fuzzy quantum probability: one is to introduce fuzziness into the event, whereas the probability (wave) is clear or determined, so as to obtain the quantum probability of fuzzy event; the other is to introduce fuzziness into the quantum probability, while the event is clear, so as to obtain the fuzzy quantum probability of the event. The literature [14] is dedicated to the work in the second direction, i.e. doing the work of "the fuzzification of quantum probability" with the method of fuzzy mathematics, whereas the work of the first direction will be done in this paper. This work will reform quantum theory and push quantum theory to a new stage.

In this paper, the form of the path integral of fuzzy event is established in Section 2. The quantum field theory of fuzzy event is discussed in Section 3. In Section 4, the mathematical form of membership degree amplitude of fuzzy path is discussed. Fuzzy diagram is also introduced and discussed in this Section.

\section{The Path Integral of Fuzzy Event}

As we know, quantum probability is calculated by "probability amplitude", whereas probability amplitude is calculated by "path integral". Thereby, if one attempts to create a theory about the quantum probability of fuzzy event, it is necessary to introduce fuzziness into path integration. For this, the three different probability theories about paths should be first reviewed.

In Figure 1, the point $A$ is the particle source whereas a detector is placed at point $B . \vec{r}(t)$ represents a possible path from $A$ to $B$. Then, the total probability of finding the particle at the point $B$ can be computed from the viewpoints of several different probability theories.

First, from the viewpoint of classical probability theory, if there exists a probability distribution for the momentum values of the emitted particles, the particle has a certain probability to move along the path $\vec{r}(t)$ and are observed at point $B$. This probability is denoted as $P[\vec{r}(t)]$. Then, the probability that a particle will be observed at point $B$ is

$$
P(A \rightarrow B)=\sum_{\substack{\text { all } \vec{r}(t) \\ \operatorname{from} A \text { to } B}} P[\vec{r}(t)] .
$$




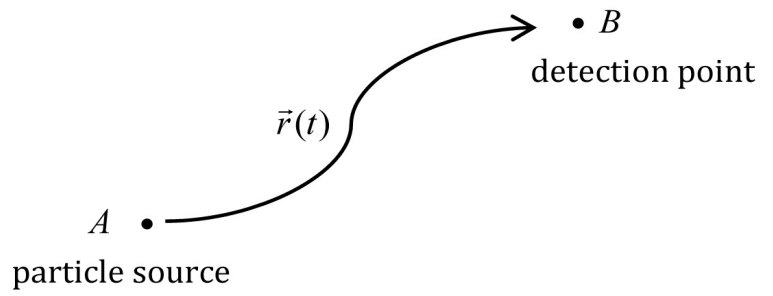

Figure 1. This figure shows the thought of path summation. For the probability theory of fuzzy event, the position (coordinate) of the particle at a certain moment is fuzzy or difficult to be judged accurately. The so-called "fuzziness of the coordinate" is different from the probability uncertainty of the coordinate, the former is the uncertainty of the judgment of the result that has occurred whereas the latter is the uncertainty of the prediction of the result that has not occurred.

Second, from the viewpoint of the probability theory of fuzzy event, considering the fuzziness of the position (coordinate) of the particle, the path of the particle also is unsharp. So, $A \rightarrow B$ is a fuzzy event, which can be denoted using a fuzzy set $\tilde{R}$. Every path belongs to this fuzzy set only in a certain degree of membership. For instance, the membership degree of the path $\vec{r}(t)$ is expressed as $\mu_{\tilde{R}}[\vec{r}(t)] \in[0,1]$. According to the probability formula of fuzzy event [15], the probability that a particle will be observed at point $B$ is

$$
P(\tilde{R}=(A \rightarrow B))=\sum_{\substack{\operatorname{all} \vec{r}(t) \\ \text { from } A \text { to } B}} \mu_{\tilde{R}}[\vec{r}(t)] \cdot P[\vec{r}(t)] .
$$

Third, from the viewpoint of quantum mechanics, considering the volatility of the particle, according to the principle of superposition, the probability amplitude for $A \rightarrow B$ is

$$
K(A \rightarrow B)=\sum_{\substack{\text { all } \vec{r}(t) \\ \operatorname{from} A \text { to } B}} \psi[\vec{r}(t)] .
$$

Here $\psi[\vec{r}(t)]$ is the probability amplitude for the path $\vec{r}(t)$. According to the statistical interpretation of wave function, the total probability should be calculated using probability amplitude

$$
P(A \rightarrow B)=|K(A \rightarrow B)|^{2} .
$$

The logic vein among these three probability theories is shown in Figure 2.

The designed path summation form about fuzzy event is also exhibited in Figure 2. Is this kind of path summation form rational? The consideration can be made as follows:

1) When the interference terms

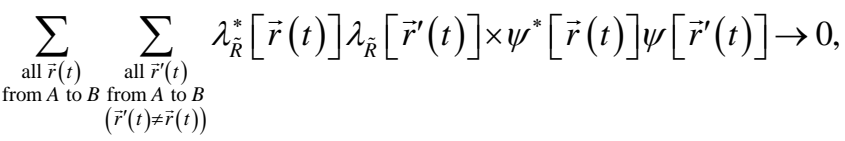

i.e. wave effect disappears, the form of the quantum probability of fuzzy event changes into

$$
P(\tilde{R}=(A \rightarrow B))=\sum_{\substack{\text { all } \vec{r}(t) \\ \operatorname{from} A \text { to } B}}\left|\lambda_{\tilde{R}}[\vec{r}(t)]\right|^{2} \times|\psi[\vec{r}(t)]|^{2} .
$$




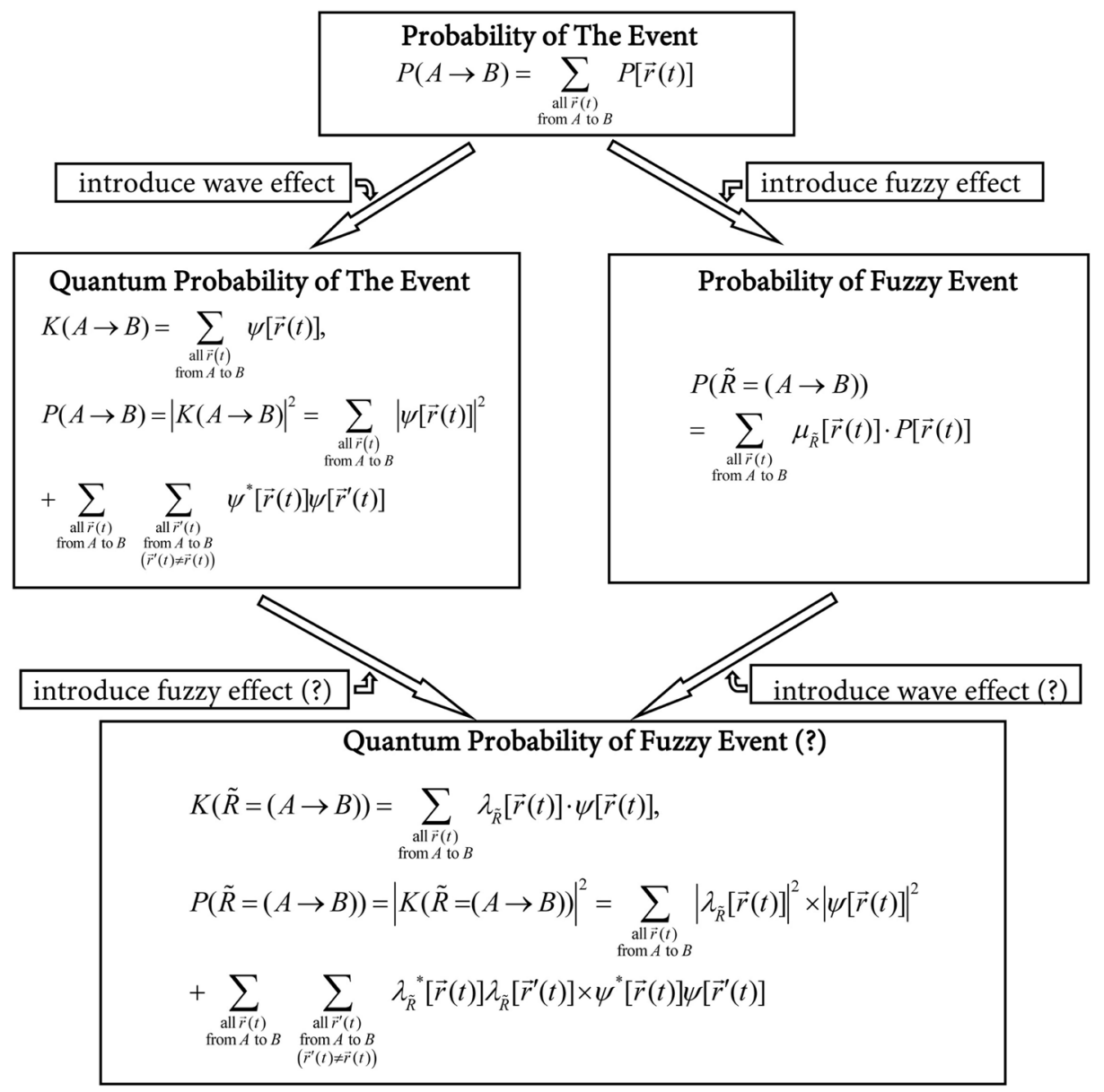

Figure 2. This figure shows the logical clues to the development of probability theory. It exhibits how fuzziness is introduced into the event. In the probability theory of fuzzy event, the property of the probability $P[\vec{r}(t)]$ of basic event has no change. Similarly, in the quantum probability theory of fuzzy event, the property of the probability amplitude $\psi[\vec{r}(t)]$ of basic event also has no change. Not only that, but the properties of the operator, eigenstate, eigenvalue etc. also are not changed. In the figure, $\left|\lambda_{\tilde{R}}[\vec{r}(t)]\right|^{2} \in[0,1]$ represents the membership degree for the path $\vec{r}(t) \in \tilde{R}$, whereas $\lambda_{\tilde{R}}[\vec{r}(t)]$ is named "membership degree amplitude" just like the origin of the name of probability amplitude. In addition, it is worth being pointed that in the probability theory of fuzzy event, the conservation of probability does not

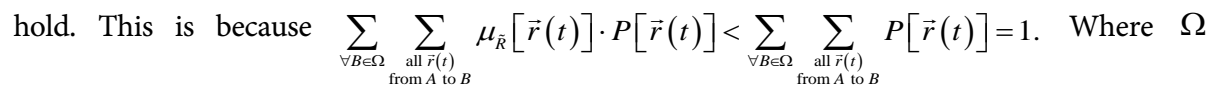
represents the whole space. Similarly, it can be imagined that in the quantum probability theory of fuzzy event, probability also is not conserved because of the introduction of the membership degree (amplitude).

This just accords with the form of the probability of fuzzy event.

2) When the membership degree amplitude

$$
\lambda_{\tilde{R}}[\vec{r}(t)] \rightarrow 1, \quad(\text { for arbitrary path) }
$$

i.e. fuzziness effect disappears, the form of the quantum probability of fuzzy event changes into 


$$
P(\tilde{R}=(A \rightarrow B))=\sum_{\substack{\text { all } \vec{r}(t) \\ \text { from } A \text { to } B}}|\psi[\vec{r}(t)]|^{2}+\sum_{\substack{\text { all } \vec{r}^{\prime}(t) \\ \text { from } A \text { to } B}} \sum_{\substack{\text { all } \operatorname{from}^{\prime}(t) \\\left(\vec{r}^{\prime}(t) \neq \bar{r}(t)\right)}} \psi^{*}[\vec{r}(t)] \psi\left[\vec{r}^{\prime}(t)\right] .
$$

This just accords with the form of the quantum probability of the event.

That is to say, under certain conditions, the quantum probability of fuzzy event can respectively transition to the probability of fuzzy event and the quantum probability of the event. So, the designed path summation form for fuzzy event in Figure 2 is rational.

According to the above thought and the explanation about Figure 2 (in the quantum probability theory of fuzzy event, the property of the probability amplitude $\psi[\vec{r}(t)]$ of basic event has no change), one can write out the path integral form of fuzzy event

$$
K\left(\left(\vec{r}_{1}, t_{1}\right) \rightarrow\left(\vec{r}_{2}, t_{2}\right)\right)=C \sum_{\text {all paths }} \lambda_{\tilde{R}}[\vec{r}(t)] \cdot \exp \{i S[\vec{r}(t)] / \hbar\},
$$

or

$$
K\left(\left(\vec{r}_{1}, t_{1}\right) \rightarrow\left(\vec{r}_{2}, t_{2}\right)\right)=\int \lambda_{\tilde{R}}[\vec{r}(t)] \cdot \exp \{i S[\vec{r}(t)] / \hbar\} D[\vec{r}(t)] .
$$

Equation (5) also can be introduced in another way. Usually, it is thought that the position (coordinate) $\vec{r}(t)$ of the particle is clear and certain at one moment. However, according to the idea of fuzzy theory [16], it can be argued that those seemingly clear and certain quantities are actually not at all clear and certain, and they have fuzziness or they are difficult to be evaluated accurately and should be depicted using fuzzy numbers. Here, it is thought that the position of the particle at one moment is fuzzy or difficult to be evaluated accurately, and it should be depicted by fuzzy number $\tilde{\vec{r}}$ (fuzzy coordinate). Thus, the range (boundary) of the coordinate value of the particle is also fuzzy. According to the integral theory of fuzzy mathematics [17], an integral of non-fuzzy function over fuzzy interval $(\tilde{a}, \tilde{b})$ can be defined

$$
\begin{aligned}
\tilde{K}\left(\left(\vec{r}_{1}, t_{1}\right) \rightarrow\left(\vec{r}_{2}, t_{2}\right)\right) & =\int_{\tilde{a}}^{\vec{b}} \exp \{i S[\vec{r}(t)] / \hbar\} D[\vec{r}(t)] \\
& =\lim _{\substack{N \rightarrow \infty \\
\varepsilon \rightarrow 0}} \int_{\tilde{x}_{1 a}}^{\tilde{x}_{1 b}} \int_{\tilde{x}_{2 a}}^{\tilde{x}_{2 b}} \cdots \int_{\tilde{x}_{N-1, a}}^{\tilde{x}_{N-1, b}} C_{N} \exp \left\{i S_{N}[\vec{r}(t)] / \hbar\right\} \prod_{j=1}^{N-1} d^{3} x_{j} .
\end{aligned}
$$

This fuzzy integral can be computed in an alternate form

$$
\begin{aligned}
K\left(\left(\vec{r}_{1}, t_{1}\right) \rightarrow\left(\vec{r}_{2}, t_{2}\right)\right) & =\lim _{\substack{N \rightarrow \infty \\
\varepsilon \rightarrow 0}} \int_{-\infty}^{\infty} \lambda_{\tilde{R}}\left(\vec{r}_{0}, \vec{r}_{1}, \cdots, \vec{r}_{N}\right) C_{N} \exp \left\{i S_{N}[\vec{r}(t)] / \hbar\right\} \prod_{j=1}^{N-1} d^{3} x_{j} \\
& =\int \lambda_{\tilde{R}}[\vec{r}(t)] \exp \{i S[\vec{r}(t)] / \hbar\} D[\vec{r}(t)] .
\end{aligned}
$$

Thus, (5) is obtained ((7) is just (5)). (7) can be regarded as the result of the non-fuzzification of (6).

Is the above design from (6) to (7) rational? An explanation can be made as follows:

From (6) and (7), it can be obtained respectively that

$$
\begin{gathered}
\left|\tilde{K}\left(\left(\vec{r}_{1}, t_{1}\right) \rightarrow\left(\vec{r}_{2}, t_{2}\right)\right)\right|^{2}=\lim _{\substack{N \rightarrow \infty \\
\varepsilon \rightarrow 0}} \int_{\tilde{x}_{1 a}}^{\tilde{x}_{1 b}} \int_{\tilde{x}_{2 a}}^{\tilde{x}_{2 b}} \cdots \int_{\tilde{x}_{N-1, a}}^{\tilde{x}_{N-1, b}}\left|C_{N} \exp \left\{i S_{N}[\vec{r}(t)] / \hbar\right\}\right|^{2} \prod_{j=1}^{N-1} \mathrm{~d}^{3} x_{j} \\
\text { + the interference terms, }
\end{gathered}
$$




$$
\begin{gathered}
\left|K\left(\left(\vec{r}_{1}, t_{1}\right) \rightarrow\left(\vec{r}_{2}, t_{2}\right)\right)\right|^{2}=\lim _{\substack{N \rightarrow \infty \\
\varepsilon \rightarrow 0}} \int_{-\infty}^{\infty}\left|\lambda_{\tilde{R}}\left(\vec{r}_{0}, \vec{r}_{1}, \cdots, \vec{r}_{N}\right) C_{N} \exp \left\{i S_{N}[\vec{r}(t)] / \hbar\right\}\right|^{2} \prod_{j=1}^{N-1} \mathrm{~d}^{3} x_{j} \\
\text { + the interference terms. }
\end{gathered}
$$

When the interference terms tend to zero (i.e. wave effect disappears), the above two equations change respectively into

$$
\begin{gathered}
\left|\tilde{K}\left(\left(\vec{r}_{1}, t_{1}\right) \rightarrow\left(\vec{r}_{2}, t_{2}\right)\right)\right|^{2}=\lim _{\substack{N \rightarrow \infty \\
\varepsilon \rightarrow 0}} \int_{\tilde{x}_{1 a}}^{\tilde{x}_{1 b}} \int_{\tilde{x}_{2 a}}^{\tilde{x}_{2 b}} \cdots \int_{\tilde{x}_{N-1, a}}^{\tilde{x}_{N-1, b}}\left|C_{N} \exp \left\{i S_{N}[\vec{r}(t)] / \hbar\right\}\right|^{2} \prod_{j=1}^{N-1} \mathrm{~d}^{3} x_{j}, \\
\left|K\left(\left(\vec{r}_{1}, t_{1}\right) \rightarrow\left(\vec{r}_{2}, t_{2}\right)\right)\right|^{2}=\lim _{\substack{N \rightarrow \infty \\
\varepsilon \rightarrow 0}} \int_{-\infty}^{\infty}\left|\lambda_{\tilde{R}}\left(\vec{r}_{0}, \vec{r}_{1}, \cdots, \vec{r}_{N}\right)\right|^{2} \times\left|C_{N} \exp \left\{i S_{N}[\vec{r}(t)] / \hbar\right\}\right|^{2} \prod_{j=1}^{N-1} \mathrm{~d}^{3} x_{j} \cdot
\end{gathered}
$$

According to the integral theory of fuzzy mathematics [17], (9) is just the alternative form of (8). This shows the rationality of (6) and (7).

\section{Quantum Field Theory of Fuzzy Event}

For the non-relativistic particle system (microscopic low-speed phenomenon) whose particle-number is certain, probability conservation is satisfied. So, there is no fuzziness, $\lambda_{\tilde{R}}[\vec{r}(t)]=1$. Thus, (5) becomes usual Feynman path integral.

But, for field theory (microscopic high-speed phenomenon), it is difficult to accurately determine the particle-number of every momentum value because of the generation and annihilation of the virtual particle pairs in vacuum. According to the fuzzy quantity theory of fuzzy mathematics [16], this kind of quantity, which is difficult to be assessed accurately, should be regarded as fuzzy variable represented by the membership function. That's to say, the microscopic vacuum state of field theory is unsharp (uncertain). It is also known that the determined microscopic state corresponds to the determined field function. Thus, the fuzziness of the microscopic state of vacuum directly leads to the fuzziness of the field function of vacuum (this can be called "fuzzy vacuum" or "fuzziness of vacuum"). Whereas the fuzziness of field function again leads to the fuzziness of the path integral of field theory. So, if (5) is used to describe field system, one can have $0 \leq|\lambda[\varphi(x)]|^{2} \leq 1$ (here real scalar field $\varphi(x)$ is taken as an example).

Here it should be pointed out that if (6) and (7) are used to describe the field theory, (6) is based on the fuzziness of the field function and fuzzy field function should be written as

$$
\tilde{\varphi}(x)=\frac{1}{(2 \pi)^{3}} \int_{-\infty}^{\infty} \tilde{q}(\vec{k}, t) \mathrm{e}^{i \vec{k} \cdot \bar{x}} \mathrm{~d}^{3} k .
$$

Where $\tilde{q}(\vec{k}, t)$ indicates the fuzziness of microscopic state of the field. Whereas in (7), since (7) is the result of the non-fuzzification of (6), the field function $\varphi(x)$ also should be regarded as the result of the non-fuzzification of $\tilde{\varphi}(x)$ with the form

$$
\varphi(x)=\left(1 /(2 \pi)^{3}\right) \int_{-\infty}^{\infty} \lambda(\vec{k}) q(\vec{k}, t) \mathrm{e}^{i \vec{k} \cdot \vec{x}} \mathrm{~d}^{3} k
$$


Where $q(\vec{k}, t)$ is a possible value of $\tilde{q}(\vec{k}, t)$, whose membership degree is maximum. $\lambda(\vec{k})$ is the relative membership degree amplitude of $q(\vec{k}, t)$, and $\mu(\vec{k})=|\lambda(\vec{k})|^{2}$ represents the relative membership degree for $q(\vec{k}, t)$. For the non-vacuum field (the field of excited state), there is no fuzziness, $\mu(\vec{k})=1$; whereas for vacuum field (the field of ground state), there is fuzziness, $0 \leq \mu(\vec{k}) \leq 1$. Here it is might as well supposed temporarily that

$$
\lambda(\vec{k})=\sqrt{\Lambda^{2 n} /\left(\vec{k}^{2}+\omega_{\vec{k}}^{2}+\Lambda^{2}\right)^{n}} .
$$

This is a form satisfying the requirement of theoretical relativistic invariance. $\Lambda(\Lambda \in R)$ and $n(n \in N)$ are two undetermined parameters (constants). Of course, besides of (11), $\lambda(\vec{k})$ or $\mu(\vec{k})=|\lambda(\vec{k})|^{2}$ also can take other forms (see Figure 4).

The introduce of $\lambda_{\tilde{R}}[\varphi(x)]$ into path integral of field theory will lead to the change of the forms of Green function and physical quantity. It is easy to prove that the expressions of the n-point Green function and physical quantity, which are corresponding to the fuzzy path integral, respectively are

$$
\begin{aligned}
G\left(x_{1}, \cdots, x_{n}\right) & =\left\langle 0\left|T\left\{\lambda_{\tilde{R}}\left[\varphi\left(x_{1}\right)\right] \hat{\varphi}\left(x_{1}\right) \cdots \lambda_{\tilde{R}}\left[\varphi\left(x_{n}\right)\right] \hat{\varphi}\left(x_{n}\right)\right\}\right| 0\right\rangle \\
& =\left.i^{-n} \frac{\delta^{n} W_{F}[J]}{\delta J\left(x_{1}\right) \cdots \delta J\left(x_{n}\right)}\right|_{J=0}, \\
\langle O\rangle & =\frac{1}{Z} \int D \varphi O[\varphi(x)] \lambda_{\tilde{R}}[\varphi(x)] \exp \left\{\frac{i}{\hbar} S[\varphi(x)]\right\} .
\end{aligned}
$$

Where $W_{F}[J]$ is the generating functional based on the fuzzy path integral, $O[\varphi(x)]$ is the physical quantity under study, and $Z$ is the fuzzy path integral about $\varphi(x)$ and its form is like (5).

It is worth being pointed out that in the quantum probability theory of fuzzy event, since probability conservation is broke, unitarity does not hold (because unitarity means probability conservation). Because unitarity also implies gauge invariance and information conservation, the breaking of unitarity will mean the breaking of gauge invariance and information (entropy) conservation. The unconservation of information (entropy) further means irreversibility. The breaking degree of unitarity can be depicted by the rate of the change of information (entropy): the change rate being large (corresponding to irreversible process) means large breaking degree, the change rate being small implies less breaking degree, and the change rate tending to zero (corresponding to reversible process) means that unitarity is not broken. Traditional quantum field theory, which argues unitarity, is applicable to the reversible process; whereas quantum field theory of fuzzy event in this paper, which negates unitarity, is suitable for the irreversible process.

The relations among these several conceptions are shown in Figure 3. 


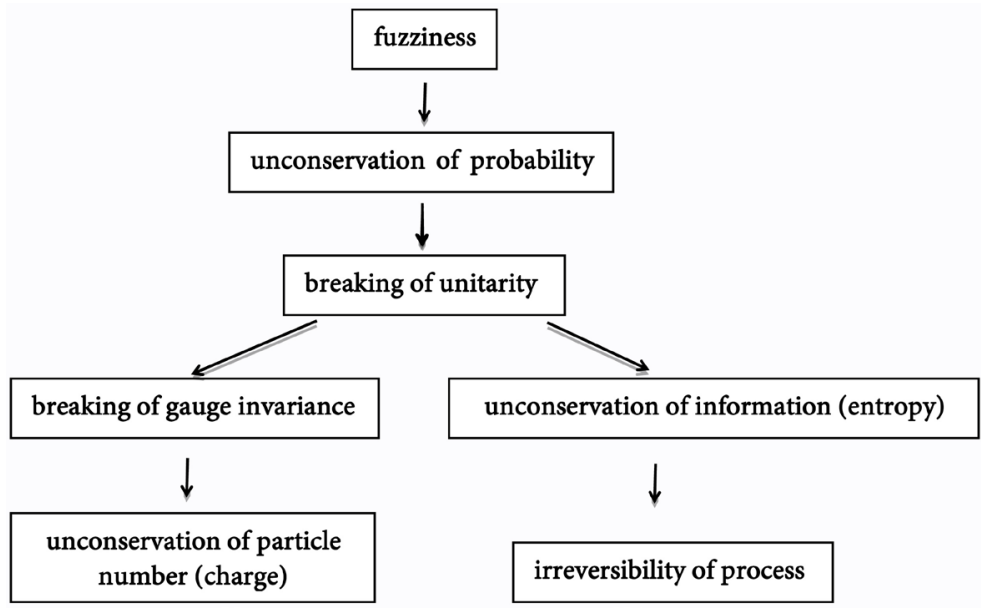

Figure 3. The breaking degree of unitarity can be depicted by such several approaches: 1) the rate of change of information (entropy), $\mathrm{d} I / \mathrm{d} t \rightarrow 0$, unitarity comes into true. 2 ) the breaking degree of gauge invariance, $k_{\mu} \Pi_{\mu \nu}(k) \rightarrow 0$ (here $\Pi_{\mu v}(k)$ is taken as an example), unitarity comes into true. 3 ) the breaking degree of probability conservation, $\left|P_{\text {sum }}-1\right| \rightarrow 0$, unitarity comes into true. It can been seen that the so-called "unitarity", "gauge invariance", "probability conservation", "information conservation" and "reversible process" are all not strict principles but approximate ones under certain conditions. It will be seen in the next section that for black hole, these approximate principles no longer hold!

\section{The Mathematical Form of Membership Degree Amplitude and Fuzzy Diagram}

In the path integral of fuzzy event, what is the mathematical form of the membership degree amplitude $\lambda_{\tilde{R}}[\varphi(x)]$ ? This is a process of exploration.

For instance, for the scattering problem of the electron-photon interaction system, it can be ordered that

$$
\begin{aligned}
& \lambda_{\tilde{R}_{1}}\left[\bar{\psi}(x), \psi(x), A_{\mu}(x)\right] \\
& =F_{1} \exp \left\{\frac{i}{\hbar} \int d^{4} x\left[\delta m \bar{\psi}(x) \psi(x)+\frac{1}{2} \delta \mu_{A}^{2} A_{\mu}(x) A_{\mu}(x)\right]\right\} .
\end{aligned}
$$

Where $d^{4} x=d x_{1} d x_{2} d x_{3} d x_{4}, \quad x_{4}=i t$ is virtual time. $F_{1}$ is a constant satisfying

$$
\begin{gathered}
0 \leq\left|\lambda_{\tilde{R}_{1}}\left[\bar{\psi}(x), \psi(x), A_{\mu}(x)\right]\right|^{2} \leq 1 . \\
\delta m=\left.\Sigma^{*}(p)\right|_{\gamma_{v} p_{v}=i m} \cdot \delta \mu_{A}^{2} \text { satisfies } \\
-i(2 \pi)^{4} \delta_{\mu \nu} \delta \mu_{A}^{2}=\Pi_{\mu v}^{*}(k \rightarrow 0) .
\end{gathered}
$$

Since gauge invariance is broken in quantum field theory of fuzzy event, the square term of gauge field is introduced into (14). This square term breaks unitarity and probability conservation. The breaking degree is decided by the integral of this square term. When the integral is relatively large, the breaking degree of unitarity also is relatively large; when the integral is relatively small, the breaking degree of unitarity also is relatively small. When the integral tends to zero, the 
square term of the gauge field can be ignored, and gauge invariance, unitarity, probability conservation and information conservation come into true.

By using (10) and (14), a $S$ matrix element without divergence, from which the theoretical results can be computed in accordance with the experimental data, can be obtained from (12). The propagators in the $S$ matrix element have the following forms:

$$
\begin{gathered}
S_{F}(p)=\mu(p) \times \frac{i}{(2 \pi)^{4}} \frac{i \hat{p}-m}{p^{2}+m^{2}-i \varepsilon}, \quad \text { (for electron) } \\
\delta_{\mu \nu} D_{F}(k)=\mu(k) \times \frac{-i}{(2 \pi)^{4}} \frac{\delta_{\mu v}}{k^{2}-i \varepsilon} . \quad \text { (for photon) }
\end{gathered}
$$

Here $\mu(p)$ and $\mu(k)$ are the membership degree factors of the propagators. Several Feynman diagrams are drawn in Figure 4 to show the membership degrees of the vacuum propagators.

These diagrams in Figure 4 are actually called "fuzzy graphs" [18] in fuzzy mathematics. It can been seen from these fuzzy graphs that in vacuum, the higher the order of the excitation is, the lower its degree of membership is. That is to say, the farther away from the influence of non-vacuum particles the vacuum is, the smaller the membership degree of its propagator is. In this way, it can be imagined that for a vacuum without the influence of non-vacuum particles, the membership degree of its propagator should tend to zero. Thus, for the vacuum without the influence of non-vacuum particles, $n$ should be a very large value in (11). The vacuum energy (density) calculated with (10) and (11) will be a very small value, which can help to explain the problem of small cosmological constant.

Since there is no divergence in the quantum field theory of fuzzy events, the renormalization is not need (Some people think that the renormalization theory cannot be regarded as a completely correct theory even though it's supported by experiments. We should also seek a deeper understanding to explain the intrinsic nature of the agreement between the experimental results and the calculated results [19]. The quantum theory of fuzzy events may be an attempt at this understanding). Though generally renormalization is not need, the renormalization of the coupling constant can be carried out for some specific purposes, for instance, researching "asymptotic freedom".

It should be pointed out that under the condition of $\Lambda_{1}=6 \sim 10 \mathrm{~m}$ and $\Lambda_{2}<1 \times 10^{-4} \mathrm{~m}$, the above fuzzy diagrams give

$$
\delta \mu_{A}^{2} \approx 0 \text { and } k_{\mu}\left[\Pi_{\mu \nu}^{*}(k)-\Pi_{\mu \nu}^{*}(0)\right] \approx 0 \rightarrow k_{\mu} \Pi_{\mu \nu}^{*}(k) \approx-i(2 \pi)^{4} k_{\mu} \delta_{\mu v} \delta \mu_{A}^{2} \approx 0 .
$$

Thus, gauge invariance, unitarity and the conservation of probability and information come into true in an approximate sense (it is mentioned above that these principles are only approximate ones).

Similarly, for the quark-gluon system, it can be ordered that

$$
\begin{aligned}
& \lambda_{\tilde{R}_{2}}\left[\bar{\psi}(x), \psi(x), B_{\mu}^{\rho}(x), \bar{\eta}(x), \eta(x)\right] \\
& =F_{2} \exp \left\{\frac{i}{\hbar} \int d^{4} x\left[\delta m_{\psi} \cdot \bar{\psi}(x) \psi(x)+\frac{1}{2} \delta \mu_{B}^{2} \cdot B_{\mu}^{\rho}(x) B_{\mu}^{\rho}(x)+\delta \mu_{\eta}^{2} \cdot \bar{\eta}^{c}(x) \eta^{c}(x)\right]\right\} .
\end{aligned}
$$



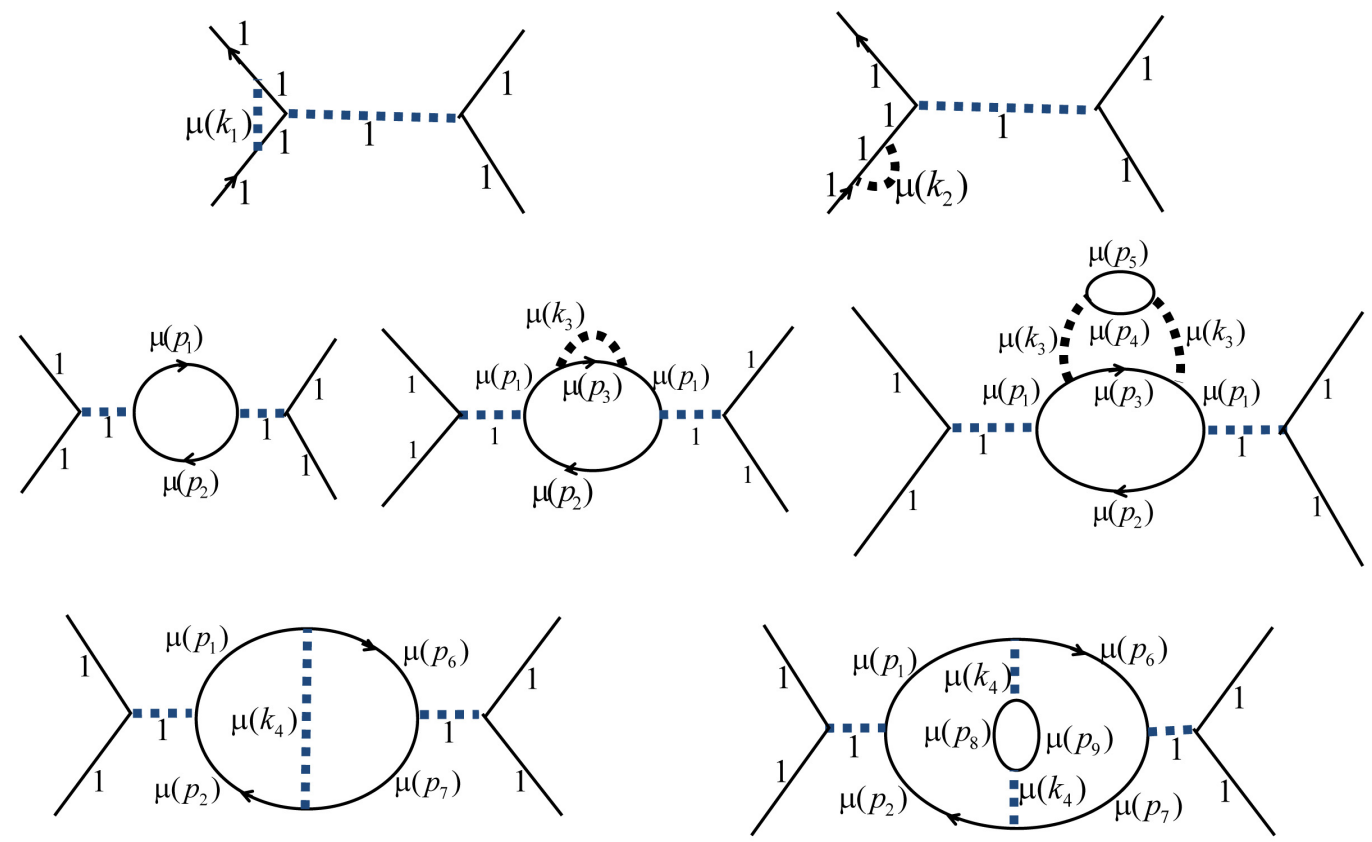

Figure 4. This figure shows the rules and laws of writing the (relative) membership degree factors of various propagators. The membership degree is assigned the value 1 for the non-vacuum propagator, $\Lambda_{2}^{2} /\left[(2 p \pm k)^{2}+\Lambda_{2}^{2}\right]$ for the vacuum electron propagator of the first-level excitation and $\left[\Lambda_{1}^{2} /\left(k^{2}+\Lambda_{1}^{2}\right)\right]^{2}$ for the vacuum photon propagator of the first-level excitation (i.e. in (11), $n=2$ ). Here, the so-called "first-level excitation" is pointing the vacuum propagator excited directly by the non-vacuum propagator. In addition, in the above digrams there are also second-level excitation, third-level excitation, fourth-level excitation, and so on. The "second-level excitation" is pointing the vacuum propagator excited directly by the vacuum propagator of the first-level excitation, and the "third-level excitation" is pointing the vacuum propagator excited directly by the vacuum propagator of the second-level excitation, and so on. In the above diagrams, the membership degrees of several vacuum propagators of the first-level excitation are respectively assigned the values as follows:

$\mu\left(k_{1}\right)=\left[\Lambda_{1}^{2} /\left(k_{1}^{2}+\Lambda_{1}^{2}\right)\right]^{2}$,

$\mu\left(k_{2}\right)=\left[\Lambda_{1}^{2} /\left(k_{2}^{2}+\Lambda_{1}^{2}\right)\right]^{2}$,

$\mu\left(p_{1}\right)=\mu\left(p_{3}\right)=\mu\left(p_{6}\right)=\Lambda_{2}^{2} /\left[\left(2 p_{1}-k\right)^{2}+\Lambda_{2}^{2}\right]$,

Where $\Lambda_{1}=6 \sim 10 \mathrm{~m}, \Lambda_{2}<1 \times 10^{-4} \mathrm{~m}, \mathrm{~m}$ is the mass of electron. For the va-

$\mu\left(p_{2}\right)=\mu\left(p_{7}\right)=\Lambda_{2}^{2} /\left[\left(2 p_{2}+k\right)^{2}+\Lambda_{2}^{2}\right]$.

cuum propagator of the second-level excitation, the assignment of the membership degree should consider the influence of the fuzziness of the vacuum propagator of the first-level excitation. For instance, in the above diagrams,

$\mu\left(k_{3}\right)=\left[\Lambda_{2}^{2} /\left(\left(2 p_{1}-k\right)^{2}+\Lambda_{2}^{2}\right)\right]\left[\Lambda_{1}^{2} /\left(k_{3}^{2}+\Lambda_{1}^{2}\right)\right]^{2}$,

$\mu\left(k_{4}\right)=\left[\Lambda_{2}^{2} /\left(\left(2 p_{1}-k\right)^{2}+\Lambda_{2}^{2}\right)\right]\left[\Lambda_{1}^{2} /\left(k_{4}^{2}+\Lambda_{1}^{2}\right)\right]^{2}$ or $\left[\Lambda_{2}^{2} /\left(\left(2 p_{2}+k\right)^{2}+\Lambda_{2}^{2}\right)\right]\left[\Lambda_{1}^{2} /\left(k_{4}^{2}+\Lambda_{1}^{2}\right)\right]^{2}$.

For the vacuum propagator of the

third-level excitation, the assignment of the membership degree should consider the influence of the fuzziness of the vacuum propagator of the second-level excitation. For instance, in the above diagrams,

$$
\begin{aligned}
& \mu\left(p_{4}\right)=\left[\Lambda_{2}^{2} /\left(\left(2 p_{1}-k\right)^{2}+\Lambda_{2}^{2}\right)\right]\left[\Lambda_{1}^{2} /\left(k_{3}^{2}+\Lambda_{1}^{2}\right)\right]^{2}\left[\Lambda_{2}^{2} /\left(\left(2 p_{4}+k_{3}\right)^{2}+\Lambda_{2}^{2}\right)\right], \\
& \mu\left(p_{5}\right)=\left[\Lambda_{2}^{2} /\left(\left(2 p_{1}-k\right)^{2}+\Lambda_{2}^{2}\right)\right]\left[\Lambda_{1}^{2} /\left(k_{3}^{2}+\Lambda_{1}^{2}\right)\right]^{2}\left[\Lambda_{2}^{2} /\left(\left(2 p_{5}-k_{3}\right)^{2}+\Lambda_{2}^{2}\right)\right], \\
& \mu\left(p_{8}\right)=\left[\Lambda_{2}^{2} /\left(\left(2 p_{1}-k\right)^{2}+\Lambda_{2}^{2}\right)\right]\left[\Lambda_{1}^{2} /\left(k_{4}^{2}+\Lambda_{1}^{2}\right)\right]^{2}\left[\left(\Lambda_{2}^{2} /\left(2 p_{8}+k_{4}\right)^{2}+\Lambda_{2}^{2}\right)\right], \\
& \mu\left(p_{9}\right)=\left[\Lambda_{2}^{2} /\left(\left(2 p_{1}-k\right)^{2}+\Lambda_{2}^{2}\right)\right]\left[\Lambda_{1}^{2} /\left(k_{4}^{2}+\Lambda_{1}^{2}\right)\right]^{2}\left[\Lambda_{2}^{2} /\left(\left(2 p_{9}-k_{4}\right)^{2}+\Lambda_{2}^{2}\right)\right] .
\end{aligned}
$$

For the vacuum propagators of the higher-level exci-

tation, and so on. 
Where $\eta(x)$ represents ghost field. But, in the case of lattice gauge field, there is no ghost field. According to the above thought, under the condition of large momentum exchange, the perturbation $S$ matrix element without divergence can be obtained whose theoretical results accord with the experimental data when $\begin{cases}\Lambda_{3} \leq 10^{-7} m_{e}, & \text { (for the membership degree factor of the vacuum propagator of gluon) } \\ \Lambda_{4} \leq 10^{-8} m_{e}, & \left.\begin{array}{l}\text { for the membership degree factor of the vacuum propagator of ghost } \\ \text { particle }\end{array}\right) \\ \Lambda_{5} \leq 10^{-9} m_{e} & \text { (for the membership degree factor of the vacuum propagator of quark) }\end{cases}$

What is more, gauge invariance, unitarity, probability conservation and information conservation are all satisfied because

$$
\begin{aligned}
& \delta \mu_{B}^{2} \approx 0 \text { and } k_{\lambda}\left[\left(\Pi^{*}\right)_{\lambda \lambda^{\prime}}^{a a^{\prime}}(k)-\left(\Pi^{*}\right)_{\lambda \lambda^{\prime}}^{a a^{\prime}}(0)\right] \approx 0 \rightarrow k_{\lambda}\left(\Pi^{*}\right)_{\lambda \lambda^{\prime}}^{a a^{\prime}}(k) \\
& \approx-i(2 \pi)^{4} k_{\lambda} \delta_{\lambda \lambda^{\prime}} f_{a b c} f_{a^{\prime} b c} \delta \mu_{B}^{2} \approx 0 .
\end{aligned}
$$

However, under the condition of extremely strong gluon field, the integral of the square term of gluon field cannot be ignored, and the breaking of unitarity also has to be considered.

Since the quantum field theory of fuzzy event need no renormalization and infinitely many offsetting terms and can naturally give meaningful results without divergence, it is the theoretical framework required for the quantization of gravity. For the system of gravity and scalar field, it is ordered that

$$
\begin{aligned}
& \lambda_{\tilde{R}_{3}}\left[h^{\mu v}(x), \bar{\theta}^{\alpha}(x), \theta_{\beta}(x), \phi(x)\right] \\
& =F_{3} \exp \left\{( i / \hbar ) \int d ^ { 4 } x \left[(1 / 2) \delta \mu_{h}^{2} h^{\mu v}(x) h_{\mu v}(x)\right.\right. \\
& \left.\left.+\delta \mu_{\theta}^{2} \bar{\theta}^{c}(x) \theta_{c}(x)+\frac{1}{2} \delta \mu_{\phi}^{2} \phi(x) \phi(x)\right]\right\} .
\end{aligned}
$$

Where $\theta(x)$ is the ghost field, $h^{\mu v}=\left(g g^{\mu v}-\eta^{\mu \nu}\right) / \kappa$ is the gravitational field. $\delta \mu_{h}^{2}$ is a very small value (this is similar to $\delta \mu_{A}^{2}$ and $\delta \mu_{B}^{2}$ above). For weak gravity system, the gravitational square term in (16) is very small and thereby can be ignored, unitarity is tenable. But, for the black hole or the universe of very early stage, due to extremely strong gravity, the gravitational square term in (16) can no longer be ignored. So, the breaking of unitarity also can no longer be ignored, and information is no longer conserved.

\section{Conclusions}

In this paper the second kind of fuzzy quantum probability theory (quantum probability of fuzzy event) is proposed. This theory can be regarded as the reformation and redevelopment of usual quantum theory, making fuzzy events also be able to be described. Under certain conditions, the quantum probability theory of fuzzy event will transition to usual quantum theory. Thereby, traditional quantum theory is only the special case of the quantum probability theory of fuzzy event. Due to the introduction of fuzziness, this new theory breaks un- 
itarity, gauge invariance, probability conservation and information conservation, making these principles become approximate ones under certain conditions. This new theory is a theory which can describe irreversible process, whereas quantum mechanics is only a theory to describe reversible process. This new theory, which needs no renormalization and can naturally give meaningful results in accordance with the experiments, is the proper theory to describe microscopic high-speed phenomenon, whereas quantum mechanics is only a proper theory to describe microscopic low-speed phenomenon.

The novelty of the work of this paper lies in: 1) bringing forward the conept and idea of "fuzzy vacuum" or "fuzziness of vacuum"; 2) putting forward that under the condition of extremely strong gauge field or gravitational field, usual quantum theory and unitarity will fail.

At present, seemly there are six directions for the quantum theory of fuzzy event to play roles:

1) Being helpful to meaningfully quantize gravity.

2) Being helpful to construct the unified theory of four basic forces.

3) Being helpful to solve the puzzle of cosmological constants.

4) Being helpful to research quantitatively the origin of the Big Bang.

5) Being helpful to understand the problem of black hole information loss.

6) Possibly providing a new idea and solution to the "abnormal" decay probability of B mesons in LHCb.

\section{Conflicts of Interest}

The author declares no conflicts of interest regarding the publication of this paper.

\section{References}

[1] Hawking, S.W. (1976) Physical Review D, 14, 2460-2472. https://doi.org/10.1103/PhysRevD.14.2460

[2] Zhang, B.C., Cai, Q.Y., You, L., et al. (2009) Physical Letters B, 675, 98-101. https://doi.org/10.1016/j.physletb.2009.03.082

[3] Zhang, B.C., Cai, Q.Y., Zhan, M.S., et al. (2011) Annals of Physics (New York), 326, 350-363. https://doi.org/10.1016/j.aop.2010.11.015

[4] Zhang, B.C., Cai, Q.Y., Zhan, M.S., et al. (2013) International Journal of Modern Physics D, 22, 1341014. https://doi.org/10.1142/S0218271813410149

[5] Hawking, S.W. (2005) Physical Review D, 72, 084013. https://doi.org/10.1103/PhysRevD.72.084013

[6] Callan, C.G. and Maldacena, J.M. (1996) Nuclear Physics B, 472, 591-608. https://doi.org/10.1016/0550-3213(96)00225-8

[7] Mathur, S.D. (2009) Classical and Quantum Gravity, 26, 224001. https://doi.org/10.1088/0264-9381/26/22/224001

[8] Page, D.N. (1993) Physics Review Letters, 71, 3743-3746. https://doi.org/10.1103/PhysRevLett.71.3743

[9] Ryu, S. and Takayanagi, T. (2006) Physics Review Letters, 96, 181602. 
https://doi.org/10.1103/PhysRevLett.96.181602

[10] Almheiri, A., Engelhardt, N., Marolf, D., et al. (2019) Journal of High Energy Physics, 2019, 63. https://doi.org/10.1007/JHEP12(2019)063

[11] Almheiri, A., Mahajan, R., Maldacena, J., et al. (2020) Journal of High Energy Physics, 2020, 13. https://doi.org/10.1007/JHEP03(2020)149

[12] Penington, G., Shenker, S.H., Stanford, D., et al. (2019) arXiv preprint arXiv: 1911. 11977

[13] Zhang, J., Hu, Y. and Zhao, Z. (2006) Modern Physics Letters A, 21, 1865-1868. https://doi.org/10.1142/S0217732306019980

[14] Qiu, W. (2020) Journal of Modern Physics, 11, 952-966. https://doi.org/10.4236/jmp.2020.116059

[15] Schwartz, D.G. (2015) On the Probability of a Fuzzy Event, with Application to Nonmonotonic Reasoning. 2015 Annual Conference of the North American Fuzzy Information Processing Society (NAFIPS) Held Jointly with 2015 th World Conference on Soft Computing (WConSC), Digipen, WA, 17-19 August 2015, 1-6. https://doi.org/10.1109/NAFIPS-WConSC.2015.7284124

[16] Timothy, J.R. (2001) Fuzzy Logic and Its Engineering Applications. Publishing House of Electronics Industry, Beijing, 75.

[17] Didier, D. and Henri, P. (1980) Fuzzy Sets and Systems Theory and Applications. Academic Press, London, 110-111.

[18] Feng, D. and Long, S. (1985) Methods and Applications of Fuzzy Mathematics. Seismological Press, Beijing, 22-24.

[19] Yin, P. (1986) The Outline of Quantum Field Theory. Shnghai Scientific \& Technical Publishers, Shanghai, 372. 